\title{
A MODEL FOR THE INTRINSIC LIMIT OF CANCER THERAPY: DUALITY OF TREATMENT-INDUCED CELL DEATH AND TREATMENT-INDUCED STEMNESS
}

\author{
A bioR $\chi$ iv PREPRINT \\ Erin Angelini ${ }^{\mathrm{a}}$, Yue Wang ${ }^{\mathrm{a}, \mathrm{b}}$, Joseph X. Zhou ${ }^{\mathrm{c}, \mathrm{d}}$, Hong Qian ${ }^{\mathrm{a}}$, and Sui Huang ${ }^{\mathrm{d}}$ \\ ${ }^{\text {a }}$ Department of Applied Mathematics, University of Washington, Seattle, WA 98195 \\ ${ }^{\mathrm{b}}$ Institut des Hautes Études Scientifiques, 91440 Bures-sur-Yvette, France \\ ${ }^{\mathrm{c}}$ Immuno-Oncology Department, Novartis Institutes for BioMedical Research, Cambridge, MA 02139 \\ ${ }^{\mathrm{d} I n s t i t u t e}$ for Systems Biology, Seattle, WA 98109
}

November 2, 2021

Conflict of interest: The authors declare no conflict of interest.

Corresponding author: Sui Huang, shuang@ systemsbiology.org

Author contributions: J.X.Z., H.Q., and S.H. designed research; E.A. and Y.W. performed research; E.A. and S.H. wrote the paper.

\begin{abstract}
Intratumor cellular heterogeneity and non-genetic cell plasticity in tumors pose a recently recognized challenge to cancer treatment. Because of the dispersion of initial cell states within a clonal tumor cell population, a perturbation imparted by a cytocidal drug only kills a fraction of cells. Due to dynamic instability of cellular states the cells not killed are pushed by the treatment into a variety of functional states, including a "stem-like state" that confers resistance to treatment and regenerative capacity. This immanent stress-induced stemness competes against cell death in response to the same perturbation and may explain the near-inevitable recurrence after any treatment. This doubleedged-sword mechanism of treatment complements the selection of preexisting resistant cells in explaining post-treatment progression. Unlike selection, the induction of a resistant state has not been systematically analyzed as an immanent cause of relapse. Here, we present a generic elementary model and analytical examination of this intrinsic limitation to therapy. We show how the relative proclivity towards cell death versus transition into a stem-like state, as a function of drug dose, establishes either a window of opportunity for containing tumors or the inevitability of progression following therapy. The model considers measurable cell behaviors independent of specific molecular pathways and provides a new theoretical framework for optimizing therapy dosing and scheduling as cancer treatment paradigms move from "maximal tolerated dose," which may promote therapy induced-stemness, to repeated "minimally effective doses" (as in adaptive therapies), which contain the tumor and avoid therapy-induced progression.
\end{abstract}

Keywords cancer $\cdot$ tumor heterogeneity $\cdot$ drug resistance $\cdot$ evolutionary dynamics $\cdot$ mathematical modeling 


\section{Introduction}

The single major cause of treatment failure in cancer therapy is the emergence of a treatment-resistant tumor that drives recurrence. Other than in the case of some early-stage tumors, it is tacitly accepted that relapse is inevitable during the course of drug treatment. This assumption has translated into the unquestioned practice of quantifying the efficacy of all treatments by how long one extends the time period between diagnosis and either progression, in the form of a clinical relapse, or death [1]. The former metric defines a progression-free survival (PFS) time, which quantifies not the prevention of progression, but a delay, as evident in the proportional hazard model [2]. Treatment success is therefore measured as a "shift to the right" of the decaying Kaplan-Meier curve, which represents the fraction of progression-free surviving patients in the treated cohort compared to that of the control group. The hence derived extension of median PFS time (or loosely equivalent, of the median time to progression, or TTP) has become a de facto measure for success of a therapy [3].

In theoretical and in vitro experimental models of post-treatment regrowth of a tumor cell population, the "recovery time" in which the surviving tumor cells regrow to reach the population threshold present at the onset of treatment is a biological characteristic of the tissue. It can be considered the equivalent of the clinical TTP [4].

Recurrence, or tumor cell regrowth after treatment, is generally thought to be the result of selection in a process of Darwinian somatic evolution: Given sufficient genetic variability in a sufficiently large initial (pretreatment) cell population, it is considered statistically certain (possibly as a result of increased mutation rate in cancer cells) that the population contains cells carrying genomic mutations that confer drug-resistance and stem-like traits [5, 6, 7, 8]. A single cell with such a mutation will survive the treatment and clonally expand, thus driving the tumor regrowth under treatment.

This genetic explanation implicitly acknowledges de facto inevitability of relapse for a certain set of parameters including mutation rate, cell population size and selection pressure [5, 6, 8, 9]. In addition, elimination of drug-sensitive cells by treatment releases drug-resistant cells from spatial and nutritional constraints and facilitates their proliferation, thereby creating an apparent causal link between treatment and recurrence [4, 10, 11, 12, 13].

In recent years, non-genetic cell phenotype plasticity and the resulting cell population heterogeneity has been recognized as a source of the resistant cell phenotype, which could underlie recurrence without implication of genetic mutations [8, 14, 15, 16, 17, 18, 19]. Extensive phenotypic heterogeneity within a population of cells is generated by the variability of an individual cell's biochemical state. Such non-genetic variability emanates, in part, from the ability of the cell's gene regulatory network to produce a diversity of stable gene expression patterns (attractors), resulting in multistability within a single clonal, isogenic population. Gene expression noise stochastically disperses individual cells in gene expression space, allowing them to occupy a range of these stable cell states. Because such stochasticity of gene expression causes continuous phenotype switching and equilibration of phenotypes, this type of heterogeneity is not subject to the rule of extinction of a phenotype, as is the case with genetic mutation.

The resulting phenotypic diversity of the isogenic cell population is, while also probabilistic, more prevalent than that generated by genetic mutations and it produces distinct, robust, recurrent, and biologically relevant phenotypic sub-states in clonal cell populations [20, 21, 22]. Such sub-states include mesenchymal, persister, or stem-like states, etc., as single-cell RNAseq now amply reveals [23, 24, 25, 19, 26, 27, 28, 29, 30]. Some of these states can confer resistance and are sufficiently robust to be inherited across several cell generations [22, 31]. In this manner, non-genetic probabilistic variation can also drive Darwinian selection of resistant cells, at least for a number of cell generations.

While both genetic and non-genetic variation arise in a probabilistic manner, there is a key difference. Because non-genetic variant attractor states are the result of regulatory mechanisms, they can also be directly induced by environmental signals. Such instruction to change gene expression programs in a directed manner by an external input via a deterministic (or strongly biased probabilistic) control, as opposed to selection of an undirected probabilistic internal change, plays a dominant role in a tumor's acquisition of stem-like drug-resistant cells at short time scales (days) compared to the clonal expansion of rare mutant clones [9]. Such regulated change of cell state may be part of a robust, evolved cellular defense mechanism against cellular toxins [32].

A growing body of evidence suggests that emergence of stem-like and therapy-resistant cells along with the associated changes in gene expression are induced (as opposed to selected) by the cytotoxic stress of treatment [29, 33, 34]. In other words, there is a causal biological mechanism linking treatment to stress to the stem-like phenotype. The recurring appearance of stem-cell-like gene expression programs, or "stemness," the speed of response and involvement of canonical signaling pathways that confer multidrug resistance (such as Wnt signaling-mediated upregulation of the ABC membrane pumps) collectively support the idea of stress-induced activation of preexisting xenobiotic resistance programs in cells by treatment [35, 36, 37]. 
Therefore, any cytocidal treatment may be a double-edge sword: while a one fraction of cells in the heterogeneous population is killed, another fraction of cells is induced by treatment stress to enter a stem-like or more resistant persister state - planting the seed for recurrence [8, 38]. Drug-induced resistance thus poses an intrinsic limit to curability of tumors under any treatment that involves cell stress, as is the case with chemotherapy, target-selected therapy or radiation. The role of somatic Darwinian evolution in recurrence relative to that of non-genetic cell state transitions has been analyzed using mathematical models in order to minimize selection pressure during treatment [4, 39, 5, 40, 41]. However, the intrinsic limit that induced resistance places on therapy has not been systematically evaluated.

Here we analyze a most elementary, generic, mathematical model for the conflicting processes that are inherent to cancer therapy: treatment-induced cell death and treatment-induced transition from a drug-sensitive phenotype to a drug-resistant (stem-like) one. Under this formulation, recurrence is "baked-into" the population dynamics and, depending on quantitative details, can become manifest. Over a relevant parameter space of an ordinary differential equation (ODE) model, we analytically evaluate the activity profiles of a drug in inducing cell death vs. transition to the resistant state. We quantify how these features of treatment relate to the intrinsic inevitability of recurrence, measured as TTP. We thus provide a formal survey of the consequence of the core process of non-genetic induction of resistance by treatment, irrespective of the ensuing selection and micro-environmental influences.

We find that depending on the relative susceptibilities for cell death versus induction of the resistant state there can be a non-monotonic dependence of TTP on drug dose, such that beyond a narrow optimal dose, the induction of resistance dominates and increasing treatment intensity will drastically shorten TTP. Thus, knowledge of the measurable propensities of cells to die or activate resistance mechanisms as a function of dose is critical information for optimizing therapy. Our focus on treatment-induced non-genetic tumor cell state change complements the evolutionary framework, fills a conceptual gap to help explain why it is so hard to cure advanced cancer and can be used for modeling scheduling to avoid treatment-associated progression as sought by adaptive therapy [11].

\section{Materials and Methods}

\section{Dynamical Model of Tumor Growth}

We consider an ODE model that describes the population dynamics of cancer cells that interconvert between two distinct cell states: drug-sensitive and drug-resistant. Let $x(t)=\left[x_{1}(t), x_{2}(t)\right]^{T}$ denote the population state vector, where $x_{1}(t)$ is the density of sensitive cells, and $x_{2}(t)$ is the density of resistant cells. Following the formulation given by Zhou et al., the sizes of these two populations are governed by the following linear ODE (Figure 11] [42]:

$$
\frac{d x}{d t}=A x, \quad A=\left[\begin{array}{cc}
b_{S}-d_{S}-k_{S R} & k_{R S} \\
k_{S R} & b_{R}-d_{R}-k_{R S}
\end{array}\right] .
$$

We analyze this model of ongoing treatment with the assumption that treatment acts by raising the per capita death rate of cancer cells. Herein, the parameter $b_{S}\left(b_{R}\right)$ denotes the fixed division rate of sensitive (resistant) cells, whereas $d_{S}\left(d_{R}\right)$ denotes the total death of sensitive (resistant) cells undergoing drug treatment. The parameter $k_{S R}$ denotes the transition rate at which sensitive cells become resistant, and $k_{R S}$ is the transition rate at which resistant cells become sensitive. All of these parameters are strictly positive and depend in a particular way from the drug dose as discussed below.

We use TTP - the time it takes for the tumor to surpass its pretreatment population size - as a quantitative measure of recurrence under drug treatment. In this model, we denote TTP by $t_{P}$, which is defined as

$$
t_{P}=\inf \{t>0 \mid N(t)>N(0)\},
$$

where $N(t)=x_{1}(t)+x_{2}(t)$ is the total number of cells in the tumor (Figure 1 ). The goal of this model is to understand how $t_{P}$ depends on the model parameters.

\section{Stability Analysis}

Aside from the degenerate case in which one eigenvalue of $A$ is zero, the only fixed point of the ODE in Eq. 11 is the point $x=0$ : extinction of all cancer cells. The eigenvalues $\lambda_{1,2}$ of $A$ determine the local stability of this fixed point, whereas the eigenvectors $v^{(1,2)}$ of $A$ give the primary directions of growth and/or decay in state space. In the supplementary materials, we derive general conditions on the growth and transition rates under which the origin is an unstable node, a stable node, or a saddle point (Table S1). For appropriate initial conditions, these three cases correspond to unchecked tumor growth, tumor extinction, and tumor regression followed by regrowth. The last scenario provides the simplest mathematical conception for the relapse phenomenon. 

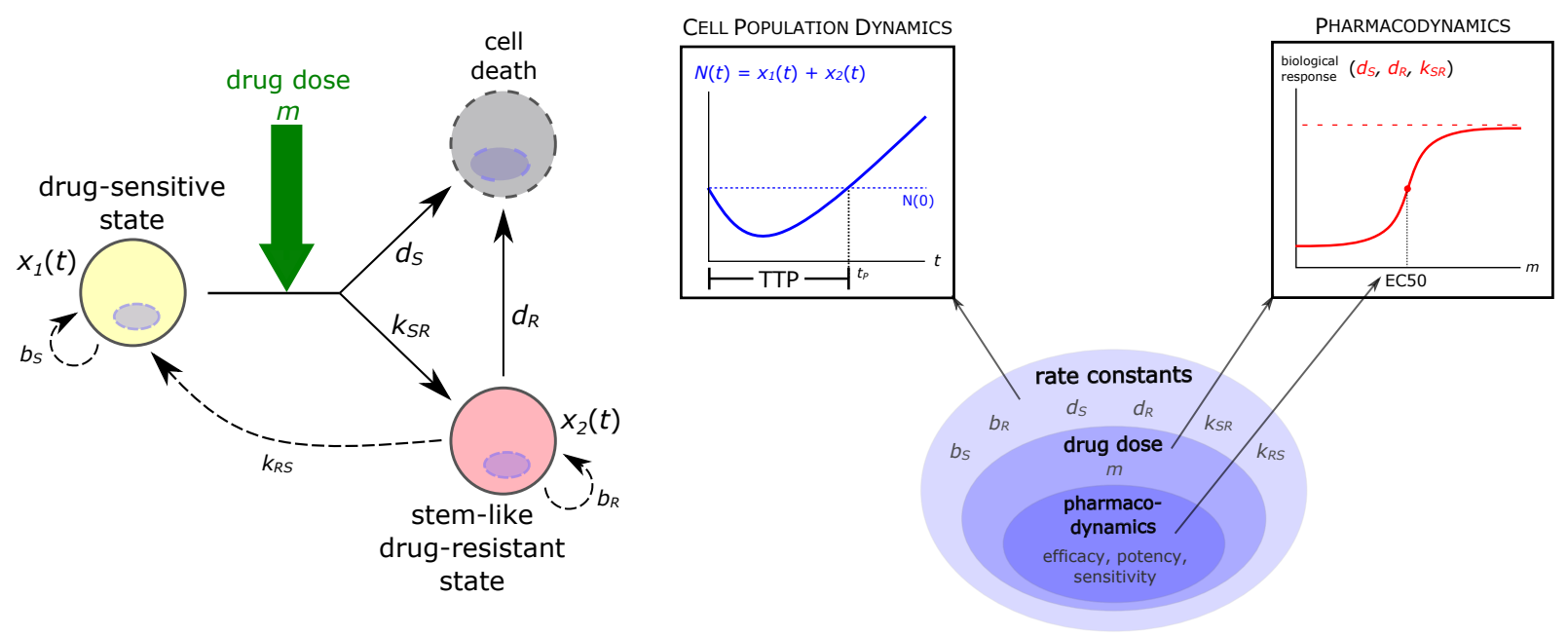

Figure 1: Levels of parameterization in the dynamical model of tumor growth. At the "highest" (i.e., most general) level, there are the rate constants that govern the growth and state transition dynamics of the cancer cell population. One level down, we introduce drug treatment into the model by assuming that the rate constants $d_{S}, d_{R}$, and $k_{S R}$ are logistic functions of drug dose $m$. The parameters that determine the shape of these dose-response curves - the pharmacodynamic constants, e.g., EC50 - form the "lowest" (i.e., most specific) level of the model parameters.

The saddle point nature of the extinction state suggests that the dynamics are ultimately difficult to control and contain. It also points to a marked "turning point" for each trajectory: if the initial tumor population is "close enough" to the stable manifold, the trajectory will first move towards the origin before being repelled away along the unstable manifold, indicating the difficulty to eradicate the tumor (Figures S2-S5). We can think of this behavior as temporary control of tumor size before the inevitable progression. Eq. S3 tells us that it is possible to control tumor regrowth wherever the relative fitness of the sensitive phenotype is sufficiently large.

The extinction state is a saddle point whenever the ratio $\left(b_{S}-d_{S}\right) /\left(b_{R}-d_{R}\right)$ is greater than the ratio $\frac{\left(b_{S}-d_{S}-k_{S R}\right)}{k_{R S}}$ (Eqs. S3). That is, temporary control of the tumor size is possible when the relative fitness of the sensitive phenotype is sufficiently large. The constant $b_{S}-d_{S}-k_{S R}$ is the net flux of the drug-sensitive population per unit density of drug-sensitive cells, and the constant $k_{R S}$ is the flux into the drug-sensitive population per unit density of drug-resistant cells. The latter parameter, sometimes referred to as the "backflow rate," is useful in characterizing how the pool of drug-resistant cells allows the drug-sensitive population to avoid extinction during chemotherapy [42].

\section{Time to Progression}

The behavior of TTP as a function of drug dose is closely related to the saddle point dynamics of the system. For one, to have $t_{P}>0$, the total population must decrease initially before recovering to its initial value, which is typically only observed when the origin is a saddle point. Moreover, under the saddle point dynamics, $t=t_{P}$ is the unique non-zero time point for which $N(t)=N(0)$.

Even with a closed expression for $N(t)$, solving the expression $N\left(t_{P}\right)=N(0)$ for $t_{P}$ is not generally possible as it involves the sum of distinct exponential terms. Instead, we can approximate $t_{P}$ by decomposing the saddle point dynamics into the distinct stages of population decrease (remission) and increase (regrowth). By definition of TTP, the total population $N(t)$ is undergoing regrowth at time $t=t_{P}$, i.e., $N^{\prime}\left(t_{P}\right)>0$. The theory of linear dynamical systems tells us that the exact solution $N(t)$ is given by a linear combination of the exponential terms $e^{\lambda_{1} t}$ and $e^{\lambda_{2} t}$, where $\lambda_{1,2}$ are the eigenvalues of $A$ (Eq. S5). In the case of a saddle point, where $\lambda_{1}>0$ and $\lambda_{2}<0$, the tumor population regrowth is necessarily driven by the exponential term corresponding to the positive eigenvalue $\lambda_{1}$. Therefore, at time $t_{P}$, we may assume that the total population is well approximated by this exponential term: $N\left(t_{P}\right) \sim e^{\lambda_{1} t_{P}}$. Under this assumption, we can approximate TTP as follows (Eqs. S5-S8):

$$
t_{P} \approx t_{P}^{*}:=\frac{1}{\lambda_{1}} \ln \left[\frac{N(0)}{c_{1}\left(v_{1}^{(1)}+v_{2}^{(1)}\right)}\right] \text {. }
$$




\section{Pharmacodynamic Model of Continuous Therapy}

To consider drug treatment, we assume that drug dose is constant throughout the course of therapy (i.e., continuous therapy) and that the rate constants $d_{S}, d_{R}$, and $k_{S R}$ depend on the amount of drug present in the system, i.e., that chemotherapy reduces tumor burden by increasing the death rate of cancer cells. At the same time, it increases the rate at which sensitive cells become resistant, the basis for the "double-edged sword" effect of chemotherapy. In mathematical terms, we introduce a secondary parameter $m$ that denotes the drug dose, and we assume that the primary parameters $d_{S}, d_{R}$, and $k_{S R}$ that capture tumor cell population dynamics are increasing functions of $m$ (Figure 11). Scaling $m$ as percentage of the maximum tolerated dose (MTD), i.e., $0 \leqslant m \leqslant 100$, we next discuss the pharmacodynamics of cellular drug response, i.e., functional forms for the dependence of the three primary parameters from $m$.

Using the commonly observed sigmoid shape of biological response curves, which reflect the cumulative probabilistic response of individual cells in a heterogeneous population, we use logistic functions to describe the rate constants $d_{S}(m), d_{R}(m)$, and $k_{S R}(m)$ (Figure 1 :

$$
\left\{\begin{array}{l}
d_{S}(m)=\delta_{S}+\frac{E_{S}}{1+\exp \left(-r_{S}\left(m-P_{S}\right)\right)} \\
d_{R}(m)=\delta_{R}+\frac{E_{R}}{1+\exp \left(-r_{R}\left(m-P_{R}\right)\right)} \\
k_{S R}(m)=\kappa+\frac{E_{S R}}{1+\exp \left(-r_{S R}\left(m-P_{S R}\right)\right)}
\end{array}\right.
$$

In the case of $d_{S}(m)$, each of the four parameters $\delta_{S}, E_{S}, r_{S}$ and $P_{S}$ determines the shape of the logistic curve and describes a behavior affected by the drug. For this reason, we refer to these parameters the pharmacodynamic parameters associated with a given rate constant for cell response. For each of the above drug responses - killing of sensitive or resistant cells and drug-induced resistance - the parameters $E, P$, and $r$ determine the efficacy, potency (EC50), and saturation rate of a given drug, respectively.

Taken together, our model incorporates three levels of parameterization: first, the parameters of the general linear population dynamics model are the rate constants $b_{S}, b_{R}$, etc. (Figure 11). Second, some of these rate constants (e.g., $k_{S R}$ ) depend on drug dose, which is represented by the parameter $m$ (Figure 1 ). And third, the way each of these rate constants depend on drug dose is determined by their respective pharmacodynamic constants, as given in Eq. 4)(Figure 11 .

In drug development implicit pharmacodynamic parameters are empirically tuned to optimize treatment outcome, which, in the case of our model, is the TTP. Teasing apart how the drug affects TTP as a function of the pharmacodynamic parameters is not a straightforward problem because we do not have an explicit expression for TTP. An exhaustive search of the entire parameter space is impractical. Because drug-induced transition to the resistant state is a hitherto unaccounted-for factor affecting recurrence, we start with a cursory analysis of how TTP changes when we vary the dose-response relationship of the sensitive-to-resistant transition rate $k_{S R}(m)$.

\section{Results}

\section{Qualitative Sensitivity Analysis}

Representative samples of the parameter space demonstrate the possible qualitatively distinct treatment outcomes - TTP as a function of drug dose - that depend on the pharmacodynamics (i.e., rate of behavior as function of drug dose $m$ ) of drug-induced resistance relative to killing (Figure 22. We define "drug resistance" by taking $E_{R} \ll E_{S}$ : the drug's cell-killing efficacy is lower for the drug-resistant phenotype than it is for the drug-sensitive phenotype. Although drug-resistance can be manifest in dampening the killing effect either by decreasing drug potency or efficacy or a combination of both, we do not vary this aspect of resistance and model resistance as the latter (lowering the maximal death rate) which reflects more profound effect on the cell state. To incorporate the observed fitness cost of resistance in the absence of drug, we further assume that $b_{S}>b_{R}$ and $\delta_{S}=\delta_{R}$ [4, 43, 44, 10].

Thus, we anchor the pharmacodynamics for killing of sensitive and resistant cells $d_{S}(m)$ and $d_{R}(m)$ and vary the parameters that determine drug-induced transition to the resistant state with respect to either EC50 $\left(P_{S R}\right)$ or efficacy $\left(E_{S R}\right)$ for the transition rate $k_{S R}(m)$ (Table S2, Figure 2).

The unknown then is how the kinetics of induction of the resistant state as a function the drug $k_{S R}(m)$ relative to the "kill curves" $d_{S}(m)$ and $d_{S}(m)$ alters TTP. The coarse-grained, but comprehensive, sensitivity analysis of our model model is thus achieved by altering the values of $P_{S R}$ and $E_{S R}$, relative to the two fixed kill curves pharmacodynamics curves (Figure 2). The following four canonical cases represent qualitatively distinct, plausible pharmacodynamical relationships between killing and induction of resistance due to treatment (Figure S1). 


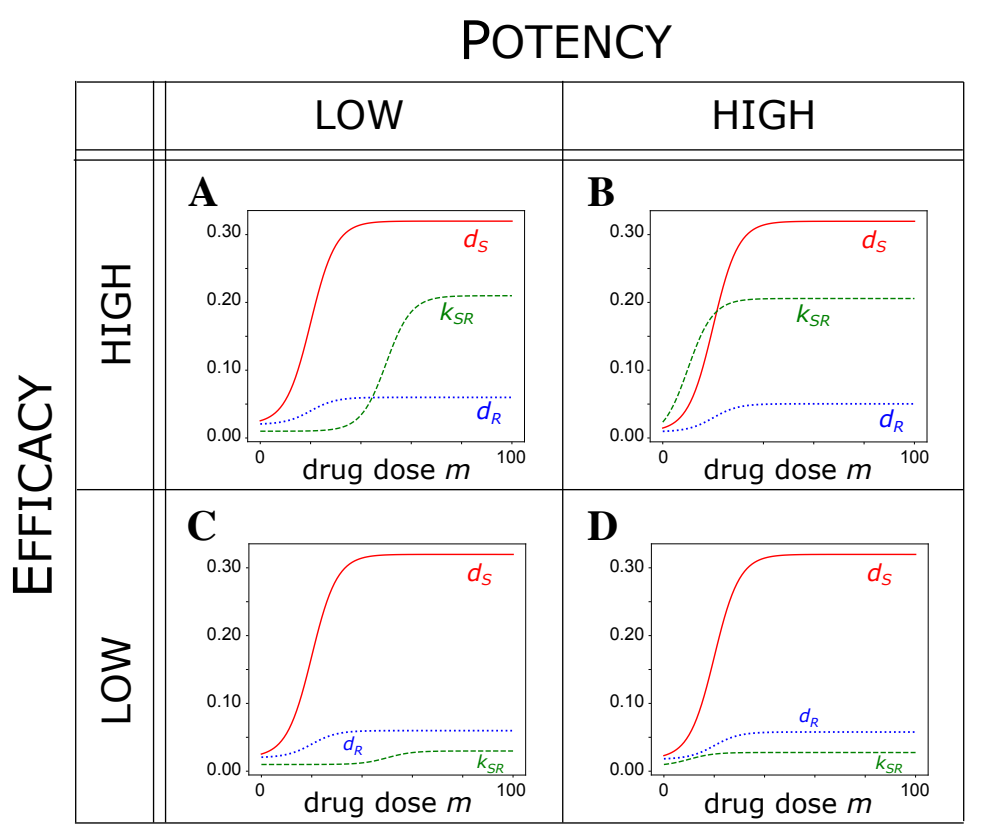

Figure 2: A coarse-grained sensitivity analysis of the effect of drug-induced resistance on treatment outcome. The parameters given in Table S2 are held fixed, while the EC50 and efficacy of resistance induction are varied in the above four cases. Efficacy increases as the parameter $E_{S R}$ increases, whereas potency decreases as the EC50 value $\left(P_{S R}\right)$ increases. A. Case A: high efficacy, low potency $\left(E_{S R}=0.2, P_{S R}=50\right)$. B. Case B: high efficacy, high potency $\left(E_{S R}=0.2, P_{S R}=10\right)$. C. Case C: low efficacy, low potency $\left(E_{S R}=0.02, P_{S R}=50\right)$. D. Case D: low efficacy, high potency $\left(E_{S R}=0.02, P_{S R}=10\right)$.

\section{Case A: high efficacy, low potency of resistance induction}

We first consider a drug with a high efficacy and low potency (i.e., high EC50) for inducing resistance to cell killing (Figure 2A). For such a drug, TTP is not monotone increasing in drug dose. Instead, it first increases before decreasing to a plateau (Figure 3A). Thus, increasing drug dose past a certain point significantly worsens the treatment outcome.

We observe that TTP increases with drug dose when an increase in dose corresponds to a significant decrease in the total growth rate relative to the total switching rate (Figure $3 \mathrm{~A}$ ). On the other hand, TTP decreases with drug dose when an increase in drug dose corresponds to a significant increase in the total switching rate relative to the total growth rate (Figure $3 \mathrm{~A}$ ). The fact that the transition dynamics dominate the growth dynamics at high drug doses indicates that under cytotoxic stress, tumor recurrence is not driven by cell growth (Figure 3). Instead, it is driven by the ability of the tumor cells to evade extinction by transitioning to a stem-like, drug-resistant state.

Returning to our analysis of the saddle point dynamics, the positive eigenvalue $\lambda_{1}$ is also non-monotonic in drug dose, first decreasing to a minimum before increasing to a plateau (Figure 3 ). This inverse relationship between $\lambda_{1}$ and TTP agrees with the above observation that $\lambda_{1}$ roughly corresponds to the rate of tumor growth during recurrence.

\section{Case B: high efficacy, high potency of resistance induction}

We now consider a drug with the same efficacy in inducing the stem-like state as in Case A but with higher potency (Figure 2B). Unlike Case A, TTP is now monotone increasing in drug dose (Figure 3B). Therefore, if the drug dose is sufficiently high, the treatment outcome in Case B is not sensitive to fluctuations about a dosage. This robustness is favorable to the sensitivity observed under Case A, in which a small fluctuation in drug dose can significantly worsen the TTP. On the other hand, the maximum TTP in Case B is low compared to that in Case A (Figure 3 A-B). A treatment that is robust to variation in drug dose is not necessarily a good one if progression is only, at most, slightly delayed.

As before, the behavior of TTP is well summarized by the positive eigenvalue $\lambda_{1}$ and the difference between the total growth and switching rates (Figure $3 \mathrm{~B}$ ). In particular, the latter shows how increased drug potency to induce stemness affects TTP. As EC50 for $k_{S R}$ is closer to that for $d_{S}$ and $d_{R}$ than it is in Case A, the total growth and state-switching rates saturate at roughly the same drug dose (Figure $3 \mathrm{~B}$ ). Therefore, the difference between the two rates 
A
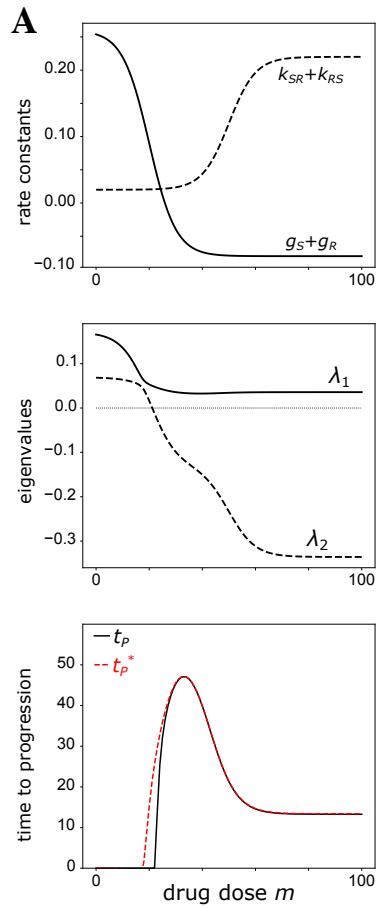

B
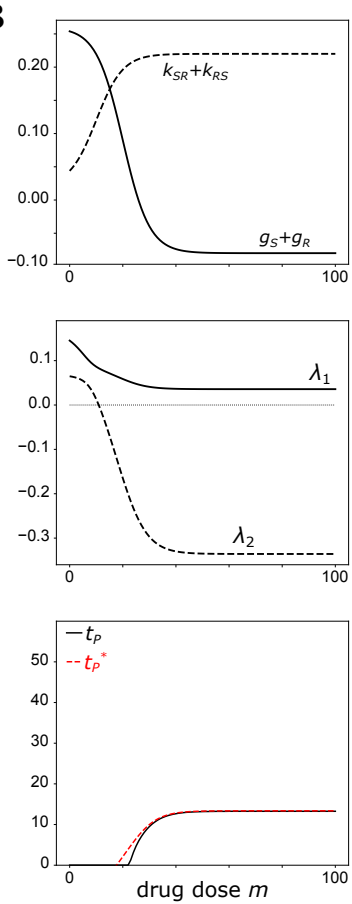

C
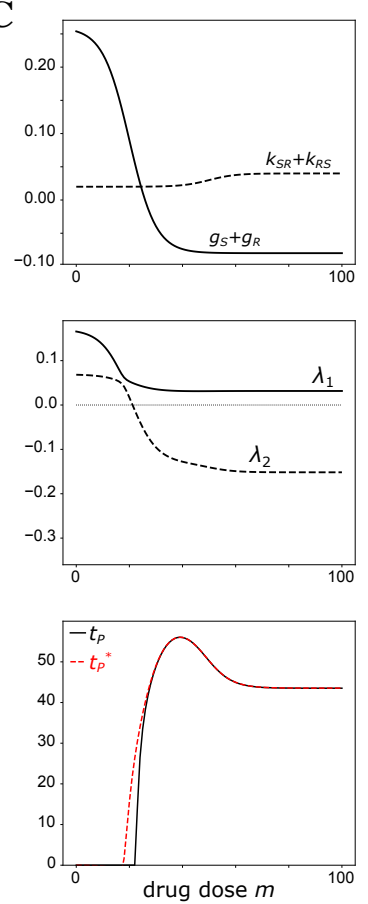

D
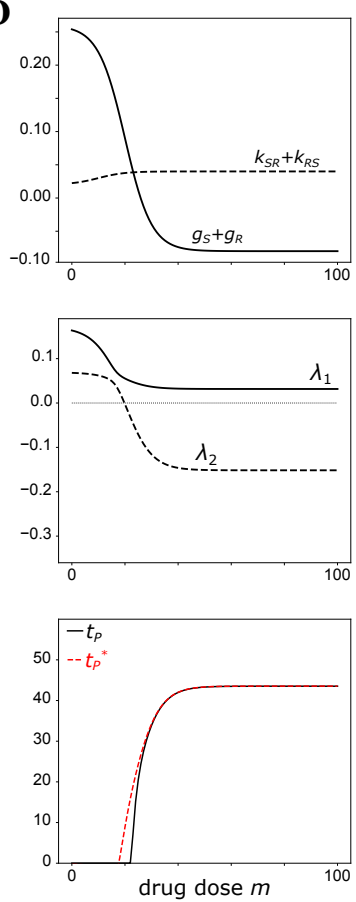

Figure 3: Summary of model behavior for Cases $\mathbf{A}, \mathbf{B}, \mathbf{C}$, and $\mathbf{D}$ as a function of drug dose $m$. The net growth rates of sensitive and resistant cells are defined as $g_{S}:=b_{S}-d_{S}$ and $g_{R}:=b_{R}-d_{R}$, respectively. First row: Total growth and transition rates, $g_{S}+g_{R}$ and $k_{S R}+k_{R S}$, respectively. Second row: Eigenvalues of the matrix $A$ in Eq. 1 . Third row: Time to progression $t_{P}$ plotted alongside its asymptotic approximation $t_{P}^{*}$.

only increases over a small range of doses, in which in TTP increases monotonically (Figure $3 \mathrm{~B}$ ). Cases A and B reveal that the relative potency of a given drug to induce resistance versus kill cells determines whether TTP is monotone increasing in drug dose or not.

\section{Case C: low efficacy, low potency of resistance induction}

For a drug with the same low potency as in Case A, but with lower efficacy, TTP is again, as in Case A, a non-monotonic function of drug dose (Figures $2 \mathrm{C}$, 3 $\mathrm{C}$ ). However, the difference between the maximum possible TTP and TTP at MTD is much smaller for the drug than in Case $\mathrm{A}$, since the maximum difference between the total growth and switching rates is smaller than in Case A (Figure $3 \mathrm{~A}, \mathrm{C}$ ). In terms of treatment outcomes, we can think of this drug as an improvement from Cases A and B: TTP is overall less sensitive to variation in drug dose than in Case A, and the maximum possible TTP is greater than that in both Cases A and B.

The overall increase in TTP is also reflected in saddle point dynamics of the system. In Case C, the magnitude of the negative eigenvalue $\lambda_{2}$ is overall lower than in Case A (Figure $3 \mathrm{C}$ ). This decrease in magnitude agrees with the increase in TTP, as $\lambda_{2}$ roughly corresponds to the rate of tumor remission in the early stages of treatment. That is, the remission stage is prolonged under treatment by a low-efficacy drug, as compared to a high-efficacy drug (Figures S2-S5).

\section{Case D: low efficacy, high potency of resistance induction}

Finally, we consider a drug that is a "combination" of Cases B and C; that is, the drug has low efficacy but high potency in inducing resistance to cell killing (Figure 2P). The TTP under this drug is a similar "combination" of Cases B and $\mathrm{C}$ : it is monotone increasing in drug dose, and its value at MTD is the same as in Case C (Figure 3P). Therefore, a drug with low efficacy and high potency for drug-induced resistance relative to killing produces a treatment scheme that effectively delays tumor progression for a wide range of drug doses. Compared to case $\mathrm{C}$, the higher potency in inducing stemness at low dose abrogates the optimal dose window, i.e., the counterintuitive "peak in TTP" at lower dose. 
A
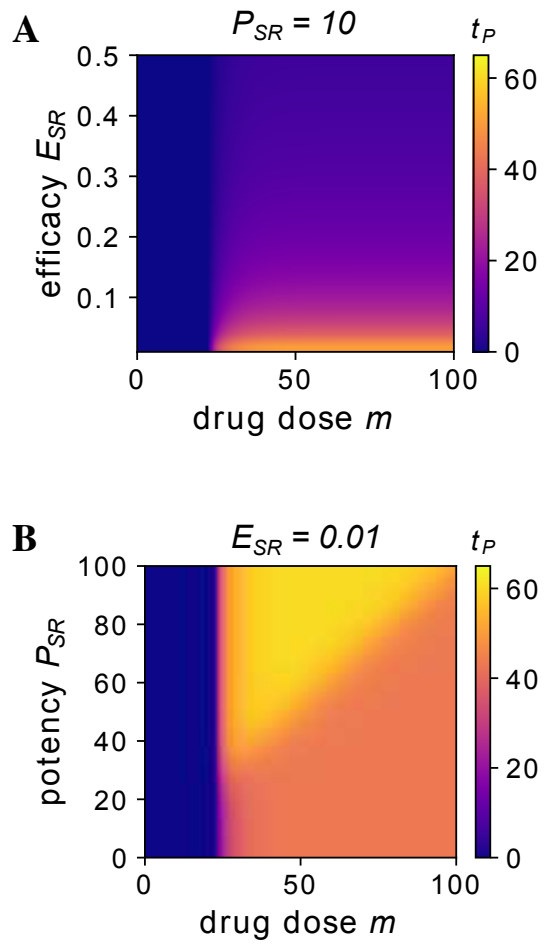

A MODEL FOR THE INTRINSIC LIMIT OF CANCER THERAPY
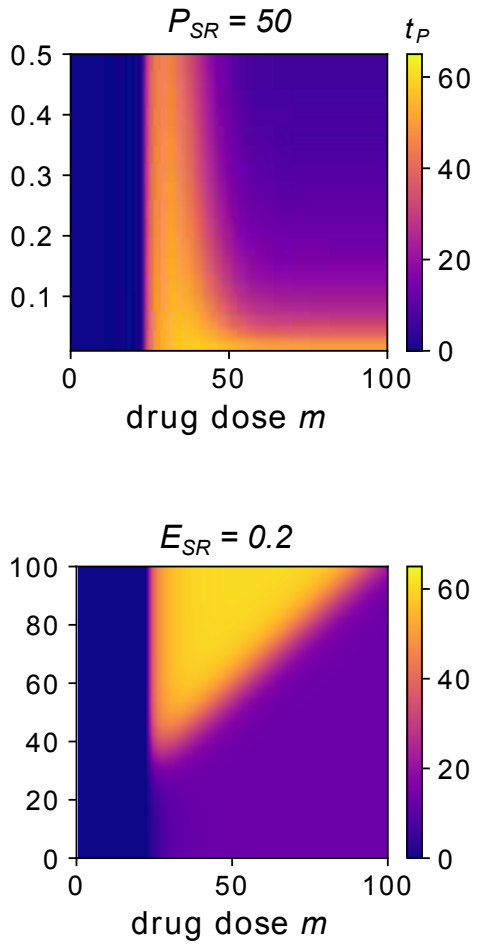
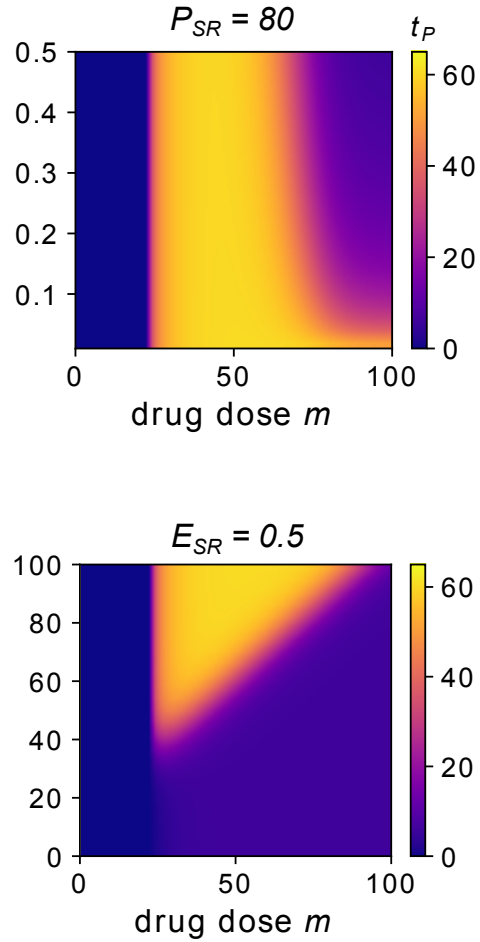

Figure 4: Time to progression $\left(t_{P}\right)$ as a function of drug dose $(m)$ plotted over a wide range of values for potency $\left(P_{S R}\right)$ and efficacy $\left(E_{S R}\right)$ of resistance induction. The value of $t_{P}$ is indicated by the colorbar on each plot. All other parameters are fixed at the values given in Table $(\mathbf{S 2})$. A. From left to right, $t_{P}$ is plotted as a function of $E_{S R}$ and $m$ for $P_{S R}=10, P_{S R}=50$, and $P_{S R}=80$. B. From left to right, $t_{P}$ is plotted as a function of $P_{S R}$ and $m$ for $E_{S R}=0.01$, $E_{S R}=0.2$, and $E_{S R}=0.5$.

\section{Parameter Search}

In order to determine how well Cases A, B, C, and D capture the dependence of TTP on drug potency $\left(P_{S R}\right)$ and efficacy $\left(E_{S R}\right)$ of resistance induction, we perform a broad parameter search. Specifically, we plot TTP as a function of drug dose over a wider range of $P_{S R}$ and $E_{S R}$ beyond the four representative cases in the main text (Figure 47. Doing so, we find that the non-monotonicity of TTP is indeed only observed when potency to induce drug resistance is low (i.e., when $P_{S R}$ is high) regardless of the value of $E_{S R}$ (Figure 44). We also find that the saturation level of TTP (i.e., its value at MTD) decreases as $E_{S R}$ increases, independent of the value of $P_{S R}$ (Figure 4B).

The results of this finer-grained parameter search indicate that the four cases given in the main text indeed characterize the dependence of TTP on the parameters $P_{S R}$ and $E_{S R}$. Thus, we can claim with certainty that the (non-)monotonicity and saturation level of TTP are respectively governed by the relative potency and efficacy to induce drug resistance. This parameter search, however, is still limited to the two parameters $P_{S R}$ and $E_{S R}$. A further parameter search, paired with rigorous sensitivity analysis, is required to characterize how TTP depends on the complete set of model parameters.

\section{Discussion}

In advanced tumors, post-treatment recurrence is almost an intrinsic feature in the course of tumor progression. It is increasingly acknowledged that even if an untreated tumor would result in rapid progression and death, recurrence after treatment is causatively or mechanistically linked to the act of treatment. The traditional explanation for recurrence after initial remission invokes selection of preexisting mutant cells in which genetic mutations confer the resistance phenotype, and more recently, the competitive release of the resistant cells, when these cells expand into the niche freed by the killing of sensitive cells due to treatment [4, 10, 11, 12, 13, 39]. 
The oft observed re-sensitization of recurrent tumors and ubiquitous cell phenotype plasticity, however, suggest a role for non-genetic, reversible phenotype switching in tumor recurrence [14, 15, 16, 17, 18, 19, 45, 46, 47]. Most recently, the induction of cells to transition into a stem-like, resilient state by the cell stress imparted by treatment has received increasing acceptance and has been confirmed by single-cell resolution measurements [20, 21, 22, 23, 24, 25, 19, 26, 27, 28, 29, 30]. Population-wide resistance to treatment induced by the same perturbation intended to kill and eradicate tumor cells poses a conflicting situation: a double-edged sword that complicates treatment response.

The mechanisms that allow a cell to be either fated to death or to enter a resistant state following the same perturbation may depend on the initial microstate due to stochastic gene expression [38]. This uncertainty is here modeled by the sigmoidal shape of the pharmacodynamical functions, which represents the cumulative probability of the dispersed propensity of cells to response in either way to treatment. We used a minimal model to characterize how the "relative strength" (potency and efficacy) of a drug to either kill tumor cells or convert them into resistance cells affects the population dynamics, as measured by the time it takes for the cell population to recover and grow to its pre-treatment size (TTP). We focused on four qualitatively distinct scenarios (A, B, C, and D) that correspond to all possible combinations of high versus low potency and high versus low efficacy.

Despite the elementary form of the model, interesting behaviors emerge: the four scenarios produced robust, prototypic behaviors for the dependence of TTP on drug dose (Figure S1). The two cases (A and C) in which the potency of the drug (dose for half-maximal effect, EC50) to induce the resistant phenotype was substantially lower than the potency to kill cells produced a non-monotonic TTP-to-drug dose relationship. In such a scenario, there is an optimal window of dose for maximal TTP: drug doses higher than the optimal dose will have lower benefits in terms of TTP.

The efficacy of the drug (the amplitude of the dose-response curve) also played a role in shaping TTP by determining the plateau value at high drug doses. High efficacy of the drug to induce the resistant state (Cases A, B) resulted, as expected, in a low value of TTP at high doses, independent of whether the dose-dependence is monotonic (Case B) or non-monotonic (Case A). On the other hand, a drug with low efficacy to induce treatment resistance (Cases C, D) resulted in a high value of TTP at high doses. Sensitivity of TTP to changes in drug efficacy means that drug dose optimization is paramount in the case where the relative potency of the drug to induce resistance vis-à-vis to kill cells is low.

Adaptive therapy, which uses the minimal effective dose - stopping treatment upon regression and re-starting if there is regrowth - would be a worthwhile treatment scheme in cases A and C. The current rationale for adaptive therapy is to avoid fixation of mutant resistant clones due to competitive release and selection [10, 11, 12, 13, 40]. However, if resistance is inducible and reversible, the intended "containment" of the tumor (as opposed to the harder-to-achieve "eradication") is even more possible than it is under the pretext of competitive release of mutant resistant clones. Thus, if we consider the new biological rationale of non-genetic, reversible dynamics of treatment-induced resistance, adaptive therapy may be even more effective than currently understood.

\section{Acknowledgements}

The authors wish to acknowledge the support of this work by the National Institutes of Health through the grants R01GM109964 and R01GM135396 and Dr. M. Strasser and Dr. A. Brock for discussions.

\section{Data availability}

Code used to generate plots in this article and in the Supporting Information can be found on GitHub (https://github.com/eeangelini/cancer-model-plots)

\section{References}

[1] S. Maetani and J. M. Gamel. Evolution of cancer survival. Surgical Oncology, 19:49-51, 2010.

[2] D. R. Cox. Regression models and life-tables. Journal of the Royal Statistical Society, 34:187-220, 1972.

[3] E. D. Saad and A. Katz. Progression-free survival and time to progression as primary end points in advanced breast cancer: often used, sometime loosely defined. Annals of Oncology, 20:460-464, 2009.

[4] J. West, Y. Ma, and P. K. Newton. Capitalizing on competition: An evolutionary model of competitive release in metastatic castration resistant prostate cancer treatment. Journal of Theoretical Biology, 455:249-260, 2018.

[5] C. S-O. Attolini and F. Michor. Evolutionary theory of cancer. Annals of the New York Academy of Sciences, 1168: 23-51, 2009. 
[6] M. Greaves and C. C. Maley. Clonal evolution in cancer. Nature, 481:306-313, 2012.

[7] L. A. Diaz Jr, R. T. Williams, J. Wu, I. Kinde, J. R. Hecht, J. Berlin, B. Allen, I. Bozic, J. G. Reiter, M. A. Novak, K. W. Kinzler, K. S. Oliner, and B. Vogelstein. The molecular evolution of acquired resistance to targeted egfr blockade in colorectal cancers. Nature, 486:537-540, 2012.

[8] N. Vasan, J. Baselga, and D.M. Hyman. A view on drug resistance in cancer. Nature, 575:299-309, 2019.

[9] A.O. Pisco and S. Huang. Non-genetic cancer cell plasticity and therapy-induced stemness in tumour relapse: 'what does not kill me strengthens me'. British Journal of Cancer, 112:1725-1732, 2015.

[10] A.F. Read, T. Day, and S. Huijben. The evolution of drug resistance and the curious orthodoxy of aggressive chemotherapy. Proceedings of the National Academy of Sciences, 108:10871-10877, 2011.

[11] J. West, L. You, J. Zhang, R.A. Gatenby, J.S. Brown, P.K. Newton, and A.R.A. Anderson. Towards multidrug adaptive therapy. Cancer Research, 80:1578-1589, 2020.

[12] J. West, R.O. Schenck, C. Gatenbee, M. Robertson-Tessi, and A.R.A. Anderson. Normal tissue architecture determines the evolutionary course of cancer. Nature Communications, 12, 2021.

[13] R. Brady-Nicholls, J.D. Nagy, T.A. Gerke, T. Zhang, A.Z. Wang, J. Zhang, R.A. Gatenby, and H. Enderling. Prostate-specific antigen dynamics predict individual responses to intermittent androgen deprivation. Nature Communications, 11, 2020.

[14] S.J. Altschuler and L.F. Wu. Cellular heterogeneity: do differences make a difference? Cell, 141:559-563, 2010.

[15] A.O. Pisco, A. Brock, J. Zhou, A. Moor, M. Mojtahedi, D. Jackson, and S. Huang. Non-darwinian dynamics in therapy-induced cancer drug resistance. Nature Communications, 4, 2013.

[16] C.L. Chaffer, I. Brueckmann, C. Scheel, A. J. Kaestli, P.A. Wiggins, L.O. Rodrigues, ..., and R.A. Weinberg. Normal and neoplastic nonstem cells can spontaneously convert to a stem-like state. Proceedings of the National Academy of Sciences, 108:7950-7955, 2011.

[17] K. Kemper, P.L. de Goeje, D.S. Peeper, and R. van Amerongen. Phenotype switching: tumor cell plasticity as a resistance mechanism and target for therapy. Cancer Research, 74:5937-5941, 2014.

[18] A. Rosech, M. Fukunaga-Kalabis, E.C. Schmidt, S.E.Zabierowski, P.A. Brafford, A. Vultur, D. Basu, P. Gimotty, T. Vogt, and M. Herlyn. A temporarily distinct subpopulation of slow-cycling melanoma cells is required for continuous tumor growth. Cell, 141:583-594, 2010.

[19] L. González-Silva, L. Quevedo, and I. Varela. Tumor functional heterogeneity unraveled by scrna-seq technologies. Trends in Cancer, 6:13-19, 2020.

[20] S. Huang, G. Eichler, Y. Bar-Yam, and D.E. Ingber. Cell fates as high-dimensional attractor states of a complex gene regulatory network. Physical Review of Letters, 94, 2005.

[21] S. Bornholdt and S. Kauffman. Ensembles, dynamics, and cell types: Revisiting the statistical mechanics perspective on cellular regulation. Journal of Theoretical Biology, 467:15-22, 2019.

[22] A. Brock, H. Chang, and S. Huang. Non-genetic heterogeneity - a mutation-independent driving force for the somatic evolution of tumors. Nature Reviews Genetics, 10:336-342, 2009.

[23] C-H. Weng, L-Y. Chen, Y-C. Lin, J-Y. Shih, Y-C. Lin, R-Y. Tseng, A-C. Chiu, Y-H. Yeh, C. Liu, Y-T. Lin, J-M. Fang, and C-C. Chen. Epithelial-mesenchymal transition (emt) beyond egfr mutations per se is a common mechanism for acquired resistance to egfr tki. Oncogene, 38:455-468, 2018.

[24] C. Neftel, J. Laffy, M.G. Filbin, T. Hara, M.E. Shore, G.J. Rahme, A.R. Richman, D. Silverbush, M.L. Shaw, C.M. Herbert, J. Dewitt, S. Gritsch, E.M. Perez, L.N. Gonzalez Castro, X. Lan, N. Druck, C. Rodman, ..., and M. Suva. An integrative model of cellular states, plasticity, and genetics for glioblastoma. Cell, 178:835-849, 2019.

[25] S.M. Shaffer, B.L. Emery, R.A. Reyes Hueros, C. Cote, G. Harmanage, D.L. Schaff, A.E. Sizemore, R. Gupte, E. Torre, A. Singh, D.S. Bassett, and A. Raj. Memory sequencing reveals heritable single-cell gene expression programs associated with distinct cellular behaviors. Cell, 182:947-959, 2020. 
[26] M.L. Suva and I. Tirosh. Single-cell rna sequencing in cancer: lessons learned an emerging challenges. Molecular Cell, 75:7-12, 2019.

[27] N.D. Marjanovic, M. Hofree, J.E. Chan, D. Canner, K. Wu, M. Trakala, ..., and T. Tammela. Emergence of a high-plasticity cell state during lung cancer evolution. Cancer Cell, 38:229-246, 2020.

[28] A. Sharma, E.Y. Cao, V. Kumar, X. Zhang, H.S. Leong, A.M. Lin, ..., and R. DasGupta. Longitudinal single-cell rna sequencing of patient-derived primary cells reveals drug-induced infidelity in stem cell hierarchy. Nature Communications, 9, 2018.

[29] Y. Su, W. Wei, L. Robert, M. Xue, J. Tsoi, A. Garcia-Diaz, B. H. Moreno, J. Kim, R. H. Ng, J. W. Lee, R. C. Koya, B. Comin-Anduix, T.G. Graeber, A. Ribas, and J.R. Heath. Single-cell analysis resolves the cell state transition and signaling dynamics associated with melanoma drug-induced resistance. Proceedings of the National Academy of Sciences, 114:13679-13684, 2017.

[30] M. Tong, Z. Deng, M. Yang, C. Xu, X. Zhang, Q. Zhang, ..., and Q. Liu. Transcriptomic but not genomic variability confers phenotype of breast cancer stem cells. Cancer Communications, 38:1-16, 2018.

[31] Y. Oren, M. Tsabar, M.S. Cuoco, L. Amir-Zilberstein, H.F. Cabanos, ..., J.S. Brugge, and A. Regev. Cycling cancer persister cells arise from lineages with distinct programs. Nature, 596:576-582, 2021.

[32] S. Huang. The logic of cancer treatment: why is it so hard to cure cancer treatment-induced progression. In B. Strauss, M. Bertolaso, I. Ernberg, and M.J. Bissell, editors, Rethinking Cancer: A New Paradigm for the Post-genomics Era, pages 63-128. MIT Press, Cambridge, MA, 2021.

[33] S.J. Wang, R. Scavetta, H.J. Lenz, K. Danenberg, P.V. Danenberg, and A.H. Schönthal. Gene amplification and multidrug resistance induced by the phosphatase-inhibitory tumor promoter, okadaic acid. Carcinogenesis, 16: 637-641, 1995.

[34] S.M. Shaffer, M.C. Dunagin, S.R. Torborg, E.A.Torre, B. Emert, ..., and A.Raj. Rare cell variability and drug-induced reprogramming as a mode of cancer drug resistance. Nature, 546:431-435, 2017.

[35] A-M. Bleau, D. Hambardzumyan, T Ozawa, E.I. Fomchenko, J.T. Huse, C. W. Brennan, and E.C. Holland. Pten/pi3k/akt pathway regulates the side population phenotype and abcg2 activity in glioma tumor stem-like cells. Cell Stem Cell, 4:226-235, 2009.

[36] S. Corrêa, R. Binato, B. Du Rocher, M.T.L. Castelo-Branco, L. Pizzatti, and E. Abdelhay. Wnt/ $\beta$-catenin pathway regulates abcb1 transcription in chronic myeloid leukemia. BMC Cancer, 12, 2012.

[37] T-H. Hung, S-C. Hsu, C-Y. Cheng, K-B. Choo, C-P. Tseng, T-C. Chen, Y-W. Lan, T-T. Huang, H-C. Lai, C-M Chen, and K-Y. Chong. Wnt5a regulates abcb1 expression in multidrug-resistant cancer cells through activation of the non-canonical pka/ $\beta$-catenin pathway. Oncotarget, 5:12273-12290, 2014.

[38] S. Huang. Genetic and non-genetic instability in tumor progression: link between the fitness landscape and the epigenetic landscape of cancer cells. Cancer Metastasis Review, 32:421-448, 2013.

[39] J.M. Greene, C. Sanchez-Tapia, and E.D. Sontag. Mathematical details on a cancer resistance model. Frontiers in Bioengineering and Biotechnology, 8, 2020.

[40] A.R.A. Anderson, A.M. Weaver, P.T. Cummings, and V. Quaranta. Tumor morphology and phenotypic evolution driven by selective pressure from the microenvironment. Cell, 127:905-915, 2006.

[41] A.P. Tran, M. A. Al-Radhawi, I. Kareva, J. Wu an D.J. Waxman, and E.D. Sontag. Delicate balances in cancer chemotherapy: modeling immune recruitment and emergence of systemic drug resistance. Frontiers in Immunology, 11, 2020.

[42] J.X. Zhou, A.O. Pisco, H. Qian, and S. Huang. Nonequilibrium population dynamics of phenotype conversion of cancer cells. PLOS One, 9, 2014.

[43] J.A. Gallaher, P.M. Enriquez-Navas, K.A. Luddy, R.A. Gatenby, and A.R.A. Anderson. Spatial heterogeneity and evolutionary dynamics modulate time to recurrence in continuous and adaptive cancer therapies. Cancer Research, 78:2127-2139, 2018. 
[44] M.A.R. Strobl, J. West, Y. Viossat, M. Damaghi, M. Robertson-Tessi, J.S. Brown, R. A. Gatenby, P.K. Maini, and A.R.A. Anderson. Turnover modulates the need for a cost of resistance in adaptive therapy. Cancer Research, 81: $1135-1147,2021$.

[45] P. Hahnfeldt and L. Hlatky. Cell resensitization during protracted dosing of heterogeneous cell populations. Radiation Research, 150:681-687, 1998.

[46] F. Fang, H. Cardenas, H. Huang, G. Jiang, S.M. Perkins, C. Zhang, H.N. Keer, Y. Liu, K.P. Nephew, and D. Matei. Genomic and epigenomic signatures in ovarian cancer associated with resensitization to platinum drugs. Cancer Research, 78:631-644, 2018.

[47] T. Liu, X. Zhang, L. Du, Y. Wang, X. Liu, H. Tian, L. Wang, P. Li, Y. Zhao, W. Duan, Y. Xie, Z. Sun, and C. Wang. Exosome-transmitted mir-128-3p increase chemosensitivity of oxaliplatin-resistant colorectal cancer. Molecular Cancer, 18, 2019. 
A MODEL FOR THE INTRINSIC LIMIT OF CANCER THERAPY

\section{Supplemental Information}

\section{Linear Stability Analysis}

By the general theory of linear ODEs, the exact solution to this system is

$$
x(t)=c_{1} e^{\lambda_{1} t} v^{(1)}+c_{2} e^{\lambda_{2} t} v^{(2)}
$$

where $\lambda_{i}, v^{(i)}$ are the right eigenpairs of $A$. The eigenvalues of $A$ are given by:

$$
\lambda_{1,2}=\frac{\alpha+2\left(g_{S}-k_{S R}\right) \pm \sqrt{\left(\alpha+2\left(g_{S}-k_{S R}\right)^{2}-4\left(g_{S} g_{R}-g_{R} k_{S R}-g_{S} k_{R S}\right)\right.}}{2}
$$

where we define the net growth rates $g_{S}=b_{S}-d_{S}$ and $g_{R}=b_{R}-d_{R}$ for simplicity.

Define $\alpha=g_{R}-g_{S}-\left(k_{R S}-k_{S R}\right)$ and $\beta=\sqrt{\alpha^{2}+4 k_{S R} k_{R S}}$. Using the expression given in Eq. S2. along with the fact that

$$
g_{S}+g_{R}-k_{S R}-k_{R S}=\alpha+2\left(g_{S}-k_{S R}\right)
$$

we can write $\lambda_{1}$ and $\lambda_{2}$ in terms of $\alpha$ and $\beta$ :

$$
\begin{aligned}
\lambda_{1,2} & =\frac{\alpha+2\left(g_{S}-k_{S R}\right) \pm \sqrt{\left(\alpha+2\left(g_{S}-k_{S R}\right)^{2}-4\left(g_{S} g_{R}-g_{R} k_{S R}-g_{S} k_{R S}\right)\right.}}{2} \\
& =\frac{\alpha+2\left(g_{S}-k_{S R}\right) \pm \sqrt{\alpha^{2}+4\left(\alpha-g_{R}\right)\left(g_{S}-k_{S R}\right)+4\left(g_{S}-k_{S R}\right)^{2}+4 g_{S} k_{R S}}}{2} \\
& =\frac{\alpha+2\left(g_{S}-k_{S R}\right) \pm \sqrt{\alpha^{2}+4\left(g_{S}-k_{S R}\right)\left(\alpha-g_{R}+g_{S}-k_{S R}\right)+4 g_{S} k_{R S}}}{2} \\
& =\frac{\alpha+2\left(g_{S}-k_{S R}\right) \pm \sqrt{\alpha^{2}-4 k_{R S}\left(g_{S}-k_{S R}\right)+4 g_{S} k_{R S}}}{2} \\
& =\frac{\alpha+2\left(g_{S}-k_{S R}\right) \pm \sqrt{\alpha^{2}+4 k_{S R} k_{R S}}}{2} \\
& =\frac{\alpha+2\left(g_{S}-k_{S R}\right) \pm \beta}{2} .
\end{aligned}
$$

Because we assume that $k_{S R}$ and $k_{R S}$ are strictly positive, the discriminant $\alpha^{2}+4 k_{S R} k_{R S}$ is strictly positive, and $\beta>0$. Therefore, both of the above eigenvalues are real and distinct. The corresponding eigenvectors are

$$
v^{(1,2)}=\frac{1}{2 k_{S R}}\left[\begin{array}{c}
-\alpha \pm \beta \\
2 k_{S R}
\end{array}\right] .
$$

The coefficients $c_{1}, c_{2}$ in Eq. S1 satisfy the linear system $x(0)=V c$, where $V$ is the matrix whose columns are the eigenvectors of $A$, and $c=\left[\begin{array}{ll}c_{1} & c_{2}\end{array}\right]^{T}$. In the following analysis, we require that $g_{R}>0$ under drug treatment as a general definition of the drug-resistant phenotype.

The origin is guaranteed to be a saddle point wherever

$$
g:=\frac{g_{S}}{g_{R}}>\frac{g_{S}-k_{S R}}{k_{R S}}
$$

The condition in Eq. S3 has two interpretations based on whether $g_{S}>0$ or $g_{S}<0$. Wherever $g_{S}>0$, Eq. S3 requires that at least one of the relative fitness of the drug-sensitive phenotype $g$ and the backflow rate $k_{R S}$ are sufficiently large compared to the net flux $g_{S}-k_{S R}$. Wherever $g_{S}<0$, Eq. S3 is true when the ratio of the net flux to the backflow rate is larger in magnitude than the relative fitness.

If instead the growth and transition rates satisfy the inequality

$$
g=\frac{g_{S}}{g_{R}}<\frac{g_{S}-k_{S R}}{k_{R S}}
$$

then the relationship between $g_{S}+g_{R}$ and $k_{S R}+k_{R S}$ determines whether the origin is stable or unstable (Table S1]. When $g_{S}+g_{R}>k_{S R}+k_{R S}$, the origin is an unstable node, and the tumor grows exponentially (Table S1). On the other hand, when $g_{S}+g_{R}<k_{S R}+k_{R S}$, the origin is a stable node, and the tumor tends toward extinction, i.e., treatment has eradicated the tumor (Table S1]. 
A MODEL FOR THE INTRINSIC LIMIT OF CANCER THERAPY

One way to frame these two cases is to consider which of the two dynamics, growth or phenotype switching, is the dominant force behind the overall population dynamics. Considering the growth of the overall population of cells, we can think of $g_{S}+g_{R}$ as the unweighted growth rate of the tumor, as the true growth rate is given by $\frac{d x_{1}}{d t}+\frac{d x_{2}}{d t}=g_{S} x_{1}+g_{R} x_{2}$. Considering only the pure phenotype switching dynamics, where total size is fixed, the quantity $k_{S R}+k_{R S}$ is the relaxation time of these dynamics. Therefore, when pure growth outweighs pure switching, tumor growth is unchecked, and no tumor control is possible. If, however, the switching dynamics dominate the underlying growth rate, the tumor will eventually be eradicated.

\section{Dynamics of Tumor Population Recovery}

The following analysis follows from the fact that we can express the total population $N(t)=x_{1}(t)+x_{2}(t)$ as a linear combination of exponential terms via the exact solution $x(t)$ :

$$
N(t)=x_{1}(t)+x_{2}(t)=c_{1}\left(v_{1}^{(1)}+v_{2}^{(1)}\right) e^{\lambda_{1} t}+c_{2}\left(v_{1}^{(2)}+v_{2}^{(2)}\right) e^{\lambda_{2} t} .
$$

We can use the exact solution given in Eq. $\mathbf{S 5}$ to derive an approximate expression for $t_{P}$, assuming that it is non-zero. If $N^{\prime}(0)<0, t=t_{P}$ is the unique non-zero time point for which $N(t)=N(0)$. Using Eq. S5, the equation $N\left(t_{P}\right)=N(0)$ reads:

$$
N\left(t_{P}\right) \equiv c_{1}\left(v_{1}^{(1)}+v_{2}^{(1)}\right) e^{\lambda_{1} t_{P}}+c_{2}\left(v_{1}^{(2)}+v_{2}^{(2)}\right) e^{\lambda_{2} t_{P}}=N(0) .
$$

Solving the above expression analytically for $t_{P}$ is not possible, as we have established that the eigenvalues $\lambda_{1,2}$ are distinct. get:

Under the assumption that the exponential term $e^{\lambda_{1} t}$ gives the dominant contribution to $N(t)$ at time $t=t_{P}$, we

$$
N\left(t_{P}\right) \approx c_{1}\left(v_{1}^{(1)}+v_{2}^{(1)}\right) e^{\lambda_{1} t_{P}}=: N^{*} .
$$

Setting the above expression equal to $N(0)$ and solving for $t_{P}$, we obtain the following approximation $t_{P}^{*}$ of $t_{P}$ :

$$
t_{P} \approx t_{P}^{*}:=\frac{1}{\lambda_{1}} \ln \left[\frac{N(0)}{c_{1}\left(v_{1}^{(1)}+v_{2}^{(1)}\right)}\right] .
$$

Taking the absolute value of the difference between $N\left(t_{P}\right)$ and its approximation $N^{*}:=N\left(t_{P}^{*}\right)$ as a proxy for the absolute error $\left|t_{P}-t_{P}^{*}\right|$, we find that the error of the approximation in Eq. $\mathrm{S7}$ scales exponentially with the negative eigenvalue $\lambda_{2}$ :

$$
\left|N\left(t_{P}\right)-N^{*}\right|=\left|c_{2}\left(v_{1}^{(2)}+v_{2}^{(2)}\right)\right| e^{\lambda_{2} t_{P}} .
$$

\section{Turning Point for Tumor Regrowth}

In the above derivation, we neglected the negative eigenvalue term to obtain a closed-form approximation for TTP. However, the rate of cancer remission is equally as important in setting TTP as is the rate of cancer regrowth. Thus, in order to better understand how $t_{P}$ changes with $m$, we also consider the point $t=t_{\min }$ at which the total population $N(t)$ reaches its global minimum. Unlike with $t_{P}$, we can determine a closed form of $t_{\min }$ in terms of the model parameters. The turning point of $N(t)$ occurs when

$$
0=\frac{d N}{d t} \equiv g_{S} x_{1}(t)+g_{R} x_{2}(t) \Longleftrightarrow \rho(t):=\frac{x_{2}(t)}{x_{1}(t)}=-\frac{g_{S}}{g_{R}} .
$$

Thus, in order to better understand the dynamics of $N(t)$, we must first understand those of the ratio $\rho(t)$ of drug-resistant cells to drug-sensitive cells.

Using the quotient rule, we can compute the derivative of this ratio, $\frac{d \rho}{d t}$ :

$$
\frac{d \rho}{d t}=\frac{x_{1} x_{2}^{\prime}-x_{2} x_{1}^{\prime}}{x_{1}^{2}}=\frac{x_{2}}{x_{1}}\left(\frac{x_{2}^{\prime}}{x_{2}}-\frac{x_{1}^{\prime}}{x_{1}}\right)=\rho\left(g_{R}-k_{R S}+\frac{k_{S R}}{\rho}-g_{S}+k_{S R}-k_{R S} \rho\right) .
$$

Recall that $\alpha=g_{R}-g_{S}-\left(k_{R S}-k_{S R}\right)$. Then the above expression simplifies to

$$
\frac{d \rho}{d t}=-k_{R S} \rho^{2}+\alpha \rho+k_{S R}
$$


This ODE is a Riccati equation with initial condition $\rho_{0}:=\rho(0)=x_{2}(0) / x_{1}(0)$. Define $q(\rho)$ as the polynomial on the right-hand side of Eq. S10.

$$
q(\rho)=-k_{R S} \rho^{2}+\alpha \rho+k_{S R} .
$$

As long as $q(\rho)$ has real roots, we can solve Eq. S10 analytically via separation of variables. Recall the definition $\beta=\sqrt{\alpha^{2}+4 k_{S R} k_{R S}}$. Then the roots of $q(\rho)$ are given by

$$
\rho_{1,2}=\frac{-\alpha \pm \beta}{-2 k_{R S}}=\frac{1}{2 k_{R S}}(\alpha \mp \beta) .
$$

We know that $\alpha^{2}+4 k_{S R} k_{R S}>0$, which tells us that the roots $\rho_{1,2}$ are real and distinct. Therefore, we can write $q(\rho)$ in the following factored form:

$$
q(\rho)=-k_{R S}\left(\rho-\rho_{1}\right)\left(\rho-\rho_{2}\right)
$$

Using the fact that $\rho_{1}<\rho_{2}$, a simple phase-line stability analysis tells us that $\rho_{1}$ is an unstable fixed point, and $\rho_{2}$ is a stable fixed point.

With this factorization of $q(\rho)$, we can now solve the given IVP to obtain a closed form of $\rho(t)$. We begin by deriving the partial fraction decomposition of $1 / q(\rho)$ :

$$
\begin{aligned}
& \frac{1}{q(\rho)}=\frac{1}{-k_{R S}\left(\rho-\rho_{1}\right)\left(\rho-\rho_{2}\right)}=\frac{C_{1}}{\rho-\rho_{1}}+\frac{C_{2}}{\rho-\rho_{2}} \\
& \Longleftrightarrow 1=-k_{R S} C_{1}\left(\rho-\rho_{2}\right)-k_{R S} C_{2}\left(\rho-\rho_{1}\right) \Longleftrightarrow-\frac{1}{k_{R S}}=\left(C_{1}+C_{2}\right) \rho-C_{1} \rho_{2}-C_{2} \rho_{1} \\
& \Longleftrightarrow C_{1}+C_{2}=0 \text { and } \frac{1}{k_{R S}}=C_{1} \rho_{2}+C_{2} \rho_{1} \\
& \Longleftrightarrow \frac{1}{k_{R S}\left(\rho_{2}-\rho_{1}\right)}=C_{1}=-C_{2} .
\end{aligned}
$$

The unique solution to the above system of linear equations for $C_{1}$ and $C_{2}$ is

$$
C_{1}=-C_{2}=\frac{1}{k_{R S}\left(\rho_{2}-\rho_{1}\right)} .
$$

Therefore, the partial fraction decomposition of $1 / q(\rho)$ is given by

$$
\frac{1}{q(\rho)}=\frac{1}{k_{R S}\left(\rho_{2}-\rho_{1}\right)}\left(\frac{1}{\rho-\rho_{1}}-\frac{1}{\rho-\rho_{2}}\right) .
$$

We can now solve the Riccati equation in Eq. S10 for $\rho(t)$ :

$$
\frac{d \rho}{d t}=q(\rho) \Longleftrightarrow \int \frac{d \rho}{q(\rho)}=\int d t,
$$

where we use the partial fraction decomposition of $1 / q(\rho)$ to evaluate the integral in $\rho$ up to a constant of integration:

$$
\int \frac{d \rho}{q(\rho)}=\frac{1}{k_{R S}\left(\rho_{2}-\rho_{1}\right)} \int\left(\frac{1}{\rho-\rho_{1}}-\frac{1}{\rho-\rho_{2}}\right) d \rho=\frac{1}{k_{R S}\left(\rho_{2}-\rho_{1}\right)} \ln \left(\frac{\rho-\rho_{1}}{\rho-\rho_{2}}\right)-C .
$$

It follows from this result that

$$
\frac{1}{k_{R S}\left(\rho_{2}-\rho_{1}\right)} \ln \left(\frac{\rho-\rho_{1}}{\rho-\rho_{2}}\right)=t+C \text {. }
$$

Applying the initial condition $\rho(0)=\rho_{0}$, we can solve for the constant $C$ :

$$
C=\frac{1}{k_{R S}\left(\rho_{2}-\rho_{1}\right)} \ln \left(\frac{\rho_{0}-\rho_{1}}{\rho_{0}-\rho_{2}}\right) .
$$

We can now solve for the exact solution $\rho$ in Eq. S11.

$$
\begin{aligned}
& \ln \left(\frac{\rho-\rho_{1}}{\rho-\rho_{2}}\right)=k_{R S}\left(\rho_{2}-\rho_{1}\right) t+\ln \left(\frac{\rho_{0}-\rho_{1}}{\rho_{0}-\rho_{2}}\right) \\
\Longleftrightarrow & \frac{\rho-\rho_{1}}{\rho-\rho_{2}}=\frac{\rho_{0}-\rho_{1}}{\rho_{0}-\rho_{2}} e^{k_{R S}\left(\rho_{2}-\rho_{1}\right)} \\
\Longleftrightarrow & \rho\left(\rho_{0}-\rho_{2}-\left(\rho_{0}-\rho_{1}\right) e^{k_{R S}\left(\rho_{2}-\rho_{1}\right)}\right)=\rho_{1}\left(\rho_{0}-\rho_{2}\right)-\rho_{2}\left(\rho_{0}-\rho_{1}\right) e^{k_{R S}\left(\rho_{2}-\rho_{1}\right)} \\
\Longleftrightarrow & \rho(t)=\frac{\rho_{1}\left(\rho_{0}-\rho_{2}\right)-\rho_{2}\left(\rho_{0}-\rho_{1}\right) e^{k_{R S}\left(\rho_{2}-\rho_{1}\right)}}{\rho_{0}-\rho_{2}-\left(\rho_{0}-\rho_{1}\right) e^{k_{R S}\left(\rho_{2}-\rho_{1}\right)}} .
\end{aligned}
$$


From this closed expression, we find an explicit expression for $t_{\min }$ by solving the equation $\rho(t)=-g_{S} / g_{R}$ :

$$
\begin{aligned}
& \rho(t)=\frac{\rho_{1}\left(\rho_{0}-\rho_{2}\right)-\rho_{2}\left(\rho_{0}-\rho_{1}\right) e^{k_{R S}\left(\rho_{2}-\rho_{1}\right)}}{\rho_{0}-\rho_{2}-\left(\rho_{0}-\rho_{1}\right) e^{k_{R S}\left(\rho_{2}-\rho_{1}\right)}}=-\frac{g_{S}}{g_{R}} \\
\Longleftrightarrow & g_{R} \rho_{1}\left(\rho_{0}-\rho_{2}\right)-g_{R} \rho_{2}\left(\rho_{0}-\rho_{1}\right) e^{k_{R S}\left(\rho_{2}-\rho_{1}\right)}=-g_{S}\left(\rho_{0}-\rho_{2}\right)+g_{S}\left(\rho_{0}-\rho_{1}\right) e^{k_{R S}\left(\rho_{2}-\rho_{1}\right)} \\
\Longleftrightarrow & \left(g_{R} \rho_{1}+g_{S}\right)\left(\rho_{0}-\rho_{2}\right)=\left(g_{R} \rho_{2}+g_{S}\right)\left(\rho_{0}-\rho_{1}\right) e^{k_{R S}\left(\rho_{2}-\rho_{1}\right)} \\
\Longleftrightarrow & \frac{\left(g_{R} \rho_{1}+g_{S}\right)\left(\rho_{0}-\rho_{2}\right)}{\left(g_{R} \rho_{2}+g_{S}\right)\left(\rho_{0}-\rho_{1}\right)}=e^{k_{R S}\left(\rho_{2}-\rho_{1}\right)} \\
\Longleftrightarrow & t_{\text {min }}:=t=\frac{1}{k_{R S}\left(\rho_{2}-\rho_{1}\right)} \ln \left(\frac{\left(g_{R} \rho_{1}+g_{S}\right)\left(\rho_{0}-\rho_{2}\right)}{\left(g_{R} \rho_{2}+g_{S}\right)\left(\rho_{0}-\rho_{1}\right)}\right)
\end{aligned}
$$

We note that in the case where $N(t)$ grows monotonically (i.e., when the origin is an unstable node), the global minimum occurs at $t=0$, so we take $t_{\min }:=0$. If $N(t)$ decays monotonically (i.e., the origin is a stable node), $t_{\min }$ is not well defined. For each of Cases $\mathrm{A}, \mathrm{B}, \mathrm{C}$, and $\mathrm{D}$, we find that as a function of drug dose, $t_{\text {min }}$ strongly correlates with TTP (Figures S1 S4). We have that $t_{P} \approx 2 t_{\min }$ at low drug doses, and $t_{P} \approx 3 t_{\text {min }}$ at high drug doses (Figures S1:S4. 


\section{Supplemental Tables and Figures}

Table S1: Summary of all possible cases for the stability of the origin under the ODE in Eq. 1.

\begin{tabular}{c|c|c} 
& $g_{S}+g_{R}>k_{S R}+k_{R S}$ & $g_{S}+g_{R}<k_{S R}+k_{R S}$ \\
\hline$g>\frac{g_{S}-k_{S R}}{k_{R S}}$ & saddle point & saddle point \\
\hline$g<\frac{g_{S}-k_{S R}}{k_{R S}}$ & unstable node & stable node
\end{tabular}

Table S2: Initial conditions, rate constants and pharmacodynamic parameters with fixed values (units not specified).

\begin{tabular}{ccc} 
Parameter & Description & Value \\
\hline$x_{1}(0)$ & concentration of sensitive cells at onset of treatment & 200 \\
$x_{2}(0)$ & concentration of resistant cells at onset of treatment & 20 \\
$b_{S}$ & per-capita birth rate of sensitive cells & 0.2 \\
$b_{R}$ & per-capita birth rate of resistant cells & 0.1 \\
$k_{R S}$ & "backflow" rate (resistant-to-sensitive) & 0.01 \\
$\delta_{S}=\delta_{R}$ & $\approx$ basal death rate of all cells & 0.02 \\
$\kappa$ & $\approx$ basal transition rate (sensitive-to-resistant) & 0.01 \\
$r_{S}=r_{R}=r_{S R}$ & drug saturation rate & 0.2 \\
$P_{S}=P_{R}$ & EC50 for cell killing & 20 \\
$E_{S}$ & drug efficacy for killing sensitive cells & 0.3 \\
$E_{R}$ & drug efficacy for killing resistant cells & 0.04 \\
\hline
\end{tabular}


A

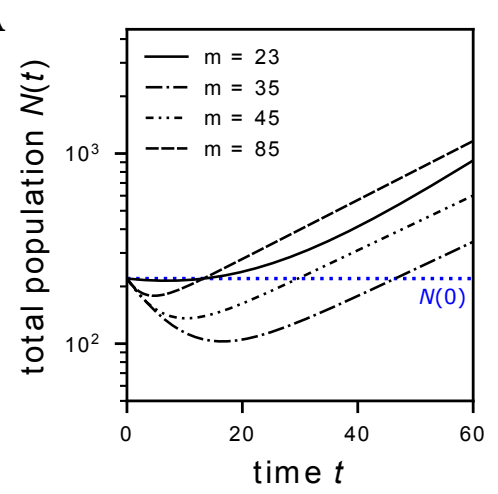

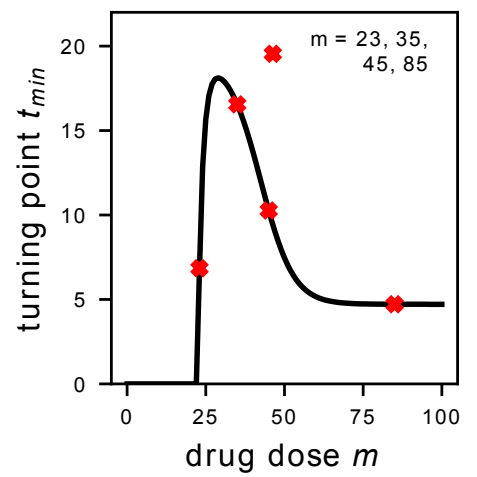

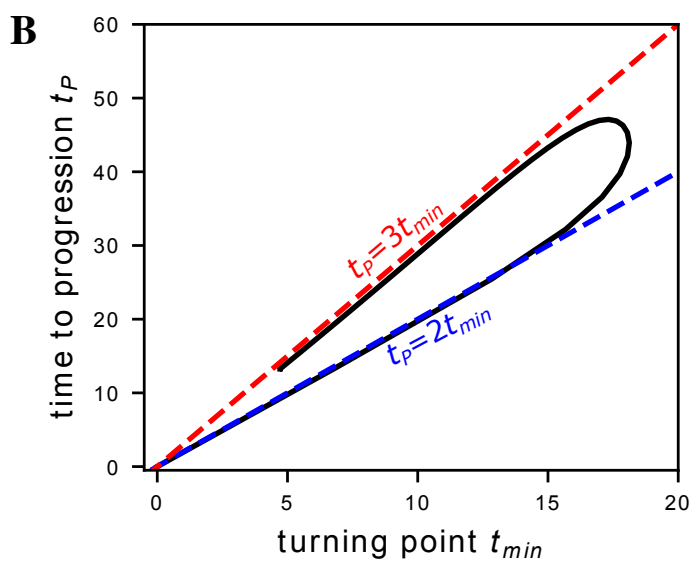

Figure S1: Dynamics of tumor population recovery for Case A. A. Left: Time-evolution of the total population $N(t)$ plotted on a logarithmic scale for drug doses $m=23,35,45,85$. The horizontal dashed line indicates initial population size $N(0)$. Right: Turning point $t_{\text {min }}$, at which $N(t)$ reaches a minimum, as a function of drug dose $m$. Red markers indicate reference points of $m=23,35,45,85$. B. Parametric curve $\left(t_{\min }(m), t_{P}(m)\right)$ relating the turning point $t_{\min }$ and the time to progression $t_{P}$. Dashed lines of slope 2 (blue) and 3 (red) are given for reference. 
A

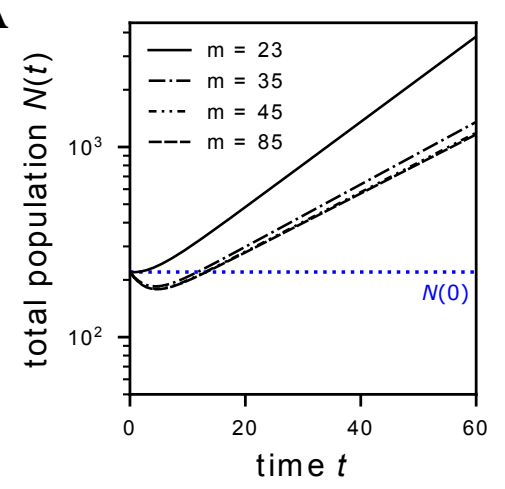

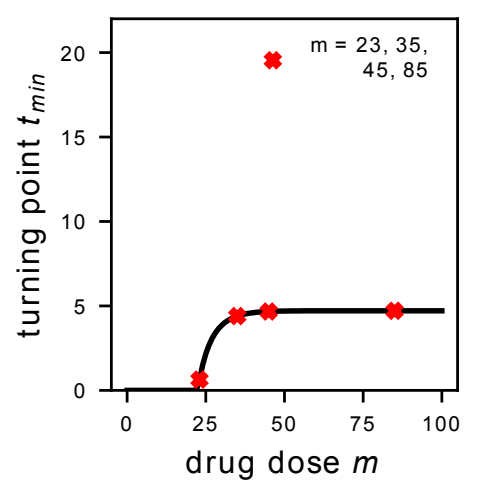

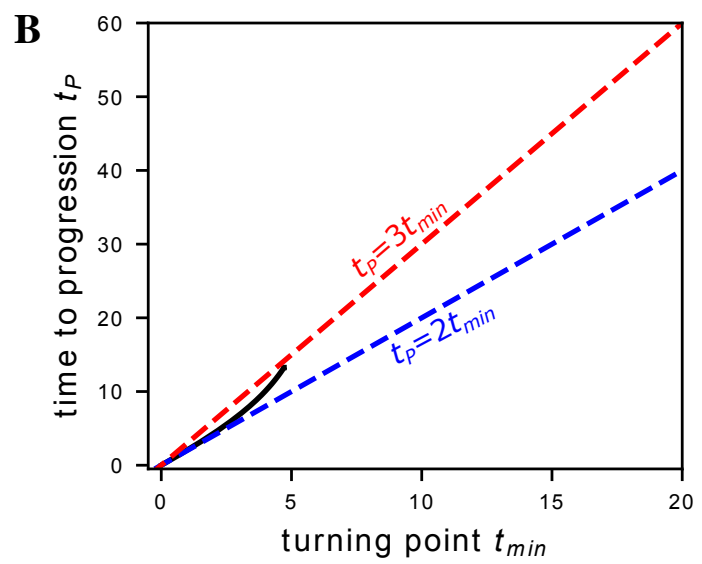

Figure S2: Dynamics of tumor population recovery for Case B. A. Left: Time-evolution of the total population $N(t)$ plotted on a logarithmic scale for drug doses $m=23,35,45,85$. The horizontal dashed line indicates initial population size $N(0)$. Right: Turning point $t_{m i n}$, at which $N(t)$ reaches a minimum, as a function of drug dose $m$. Red markers indicate reference points of $m=23,35,45,85$. B. Parametric curve $\left(t_{\min }(m), t_{P}(m)\right)$ relating the turning point $t_{\min }$ and the time to progression $t_{P}$. Dashed lines of slope 2 (blue) and 3 (red) are given for reference. 
A

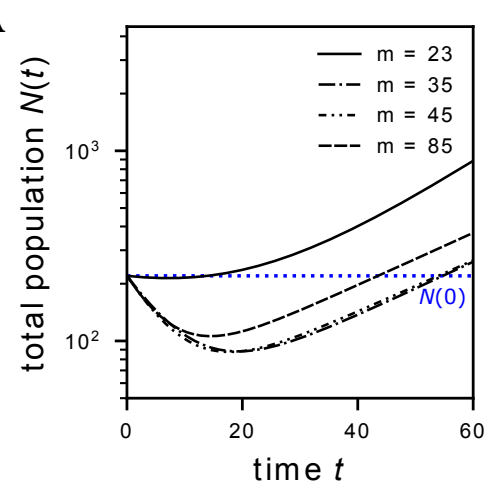

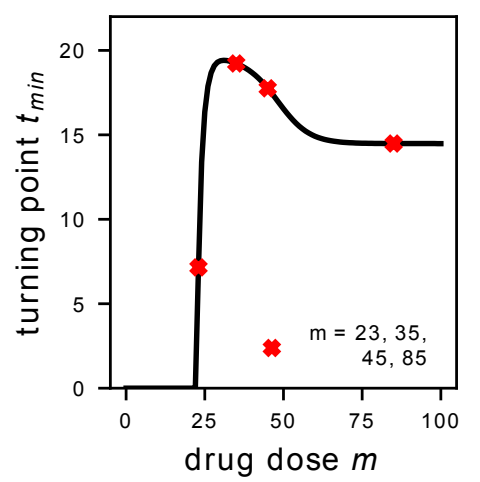

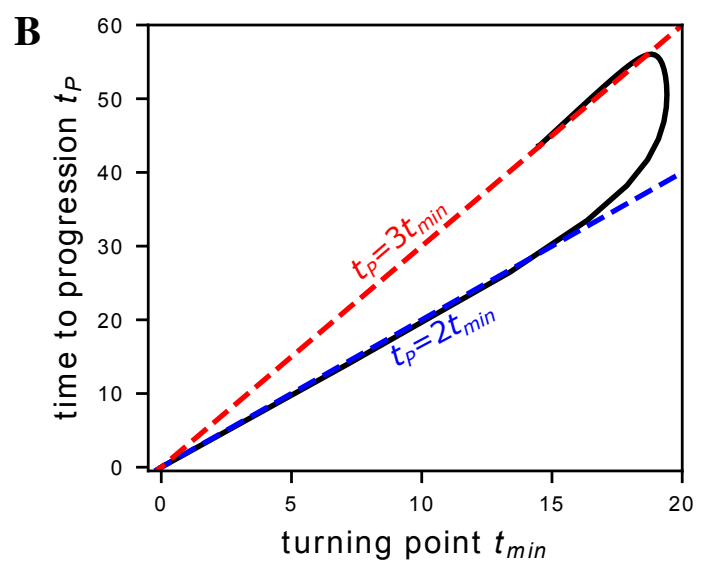

Figure S3: Dynamics of tumor population recovery for Case C. A. Left: Time-evolution of the total population $N(t)$ plotted on a logarithmic scale for drug doses $m=23,35,45,85$. The horizontal dashed line indicates initial population size $N(0)$. Right: Turning point $t_{m i n}$, at which $N(t)$ reaches a minimum, as a function of drug dose $m$. Red markers indicate reference points of $m=23,35,45,85$. B. Parametric curve $\left(t_{\min }(m), t_{P}(m)\right)$ relating the turning point $t_{\min }$ and the time to progression $t_{P}$. Dashed lines of slope 2 (blue) and 3 (red) are given for reference. 
A

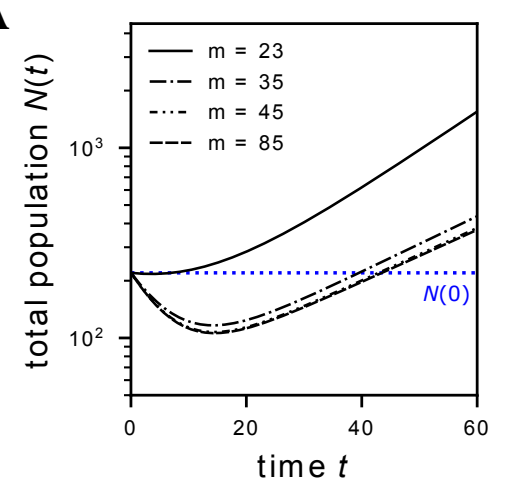

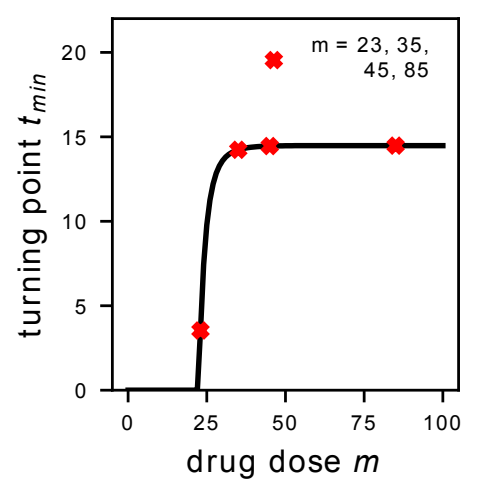

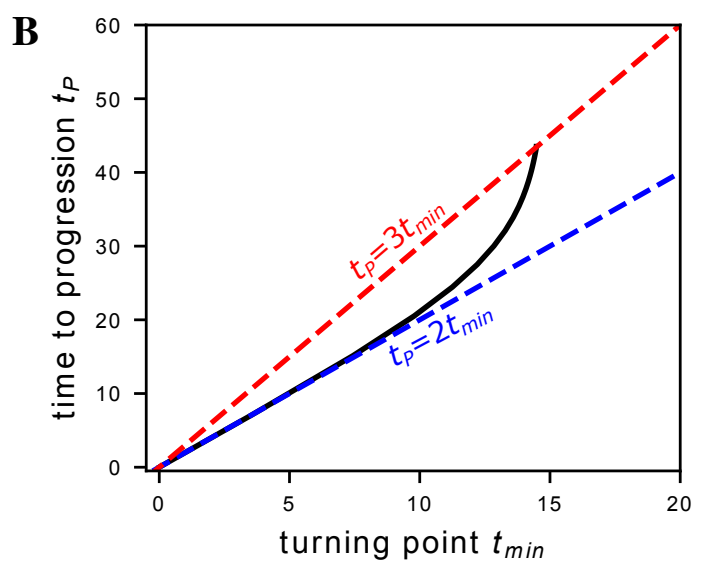

Figure S4: Dynamics of tumor population recovery for Case D. A. Left: Time-evolution of the total population $N(t)$ plotted on a logarithmic scale for drug doses $m=23,35,45,85$. The horizontal dashed line indicates initial population size $N(0)$. Right: Turning point $t_{m i n}$, at which $N(t)$ reaches a minimum, as a function of drug dose $m$. Red markers indicate reference points of $m=23,35,45,85$. B. Parametric curve $\left(t_{\min }(m), t_{P}(m)\right)$ relating the turning point $t_{\min }$ and the time to progression $t_{P}$. Dashed lines of slope 2 (blue) and 3 (red) are given for reference. 